\title{
Les technologies médiatiques dans le traitement de la crise de Grand-Bassam en Côte d'Ivoire
}

Media technologies in the treatment of the grand bassam crisis in côte d'ivoire La tecnología de los medios en el tratamiento de la crisis grand-bassam, en côte d'ivoire

Jules Evariste Agnini Toa

\section{OpenEdition}

\section{Journals}

Édition électronique

URL : http://journals.openedition.org/ctd/354

DOI : $10.4000 /$ ctd. 354

ISSN : 2491-1437

Éditeur

Chaire Unesco Pratiques émergentes en technologies et communication pour le développement

Référence électronique

Jules Evariste Agnini Toa, « Les technologies médiatiques dans le traitement de la crise de GrandBassam en Côte d'Ivoire ", Communication, technologies et développement [En ligne], 5 | 2018, mis en ligne le 04 septembre 2017, consulté le 02 mai 2019. URL : http://journals.openedition.org/ctd/354 ; DOI : $10.4000 /$ ctd.354

Ce document a été généré automatiquement le 2 mai 2019.

Communication, technologies et développement 


\title{
Les technologies médiatiques dans le traitement de la crise de Grand- Bassam en Côte d'Ivoire
}

\author{
Media technologies in the treatment of the grand bassam crisis in côte d'ivoire \\ La tecnología de los medios en el tratamiento de la crisis grand-bassam, en côte \\ d'ivoire
}

Jules Evariste Agnini Toa

\section{Introduction}

1 De nombreuses crises militaro-politiques et socioculturelles ont affecté la Côte d'Ivoire depuis la succession d'événements majeurs. On peut noter, pour rappel, le coup d'État de décembre 1999, la rébellion de septembre 2002 et la crise postélectorale de décembre 2010 à avril 2011. En outre, des crises connexes ont jalonné la vie des Ivoiriens ces derniers mois, mais c'est, indéniablement, l'attaque terroriste du 13 mars 2016 à GrandBassam, en Côte d'Ivoire, qui a fait déborder le vase. Cette crise a été relayée à grande vitesse à travers le monde grâce aux Technologies de l'Information et de la Communication (TIC) et, notamment, les technologies médiatiques, qui font l'objet de l'analyse présente.

2 Pour mener à bien cette réflexion, l'étude s'appuie sur la théorie des représentations sociales en s'inscrivant au niveau épistémologique dans la sphère de la socioanthropologie qui permet d'envisager l'étude des rapports de l'individu avec sa société, la société étant ici la Côte d'Ivoire en pleine mutation dans son vécu quotidien par les technologies médiatiques.

3 En outre, ce travail s'inscrit dans la théorie des organisations, notamment dans l'approche technologique de Joan Woodward (Woodward,1965). Les résultats des travaux de celui-ci, rappelés par Jean-Michel Plane, ont montré que «Ce sont les similitudes des systèmes technologiques et de production qui permettent d'expliquer les similitudes d'organisation 
des entreprises» (Plane, 2014, p. 52). En d'autres termes, du point de vue de l'impact des technologies médiatiques dans la crise de Grand-Bassam, les sociétés ayant été pétries dans le même moule technologique semblent avoir des modes semblables d'organisation et de diffusion des informations.

Ainsi la problématique de cette étude est de savoir s'il y a une adéquation entre l'information gouvernementale diffusée et celle perçue par la population à travers les réseaux sociaux. L'objectif est de montrer l'impact des technologies médiatiques numériques dans le traitement de la crise de Grand-Bassam et de faire l'analyse des limites de la gestion communicationnelle du gouvernement dans la diffusion de l'information.

5 L'hypothèse générale, formulée dans ce cadre est que l'appropriation des technologies médiatiques par la population a eu un impact réel à travers la production et la diffusion des informations pendant la crise terroriste de Grand-Bassam et sa réception par celle-ci.

6 Sur le plan méthodologique, il faut préciser que la base empirique de cette réflexion est une enquête qualitative de terrain conduite dans la commune de Grand-Bassam et dans le district d'Abidjan. L'échantillon qui a servi à faire le travail, comprenait des couches socioprofessionnelles, des directeurs de communication, des leaders politiques et religieux, des restaurateurs, des leaders de la société civile, différents groupes ethniques de Côte d'Ivoire, etc. Un guide d'entretien de dix questions a été administré à chacune des entités énumérées plus haut et à raison de trois personnes par entité, soit un total de douze personnes. Cette enquête a été complétée par une étude documentaire pour recueillir les opinions des internautes et analyser cinquante articles de presse (journaux ivoiriens, Jeune Afrique, AFP, etc.) et des réseaux sociaux liés à la thématique de crise de Grand-Bassam. La période du 13 mars au 13 avril 2016, soit un mois après la crise, a été retenue pour couvrir le champ de l'étude.

7 Il apparaît maintenant nécessaire d'élucider les concepts de crise et de technologie médiatique dont les données sont essentielles dans l'analyse des médias numériques et dans la transformation des habitudes culturelles des Ivoiriens. Le point relatif à la crise de communication gouvernementale pendant l'attaque de Grand-Bassam permet d'élargir le champ de réflexion pour montrer les limites de la communication gouvernementale en temps de crise. L'esquisse de quelques solutions à mettre en œuvre en matière de communication de crise achève cette réflexion.

\section{Concepts de crise et de technologie médiatique}

\section{Contexte et approche définitionnelle de la crise}

8 «"Attaque de Grand-Bassam", "attaque terroriste de Grand-Bassam", "attentat présumé à Grand- Bassam", "crise à Grand-Bassam", etc. ", tels sont les titres qui ont fait la "Une" de nombreux journaux ivoiriens au lendemain des événements douloureux qui ont secoué la cité balnéaire de Grand-Bassam située à une trentaine de kilomètres d'Abidjan. C'est une attaque à la kalachnikov qui s'est déroulée le dimanche 13 mars 2016, aux alentours de midi, à l'hôtel "Étoile du Sud » : des assaillants seraient à l'origine de ce triste événement qui s'est soldé par plusieurs morts et des blessés graves. Cette situation violente et inattendue s' est muée en une crise dans un contexte où le gouvernement ivoirien était en quête de visibilité médiatique après avoir essuyé de nombreuses difficultés selon Claude Koudou 
« liées à la naturalisation de l'ex-Président Blaise Compaoré et la question de son extradition pour répondre des crimes à lui imputés; l'affaire Guillaume Soro / Djibril Bassolé /Gilbert Diendéré ; les foudres des ONG relativement aux atteintes des droits de l'Homme $»^{1}$. par l'amélioration d'accès aux services de base, l'extension de la connectivité et la création des opportunités d'emplois, de création de richesse et de réduction de la pauvreté. De plus, les technologies médiatiques ajoutées aux médias traditionnels continuent de façonner les modes de vie des usagers. Elles jouent plus que jamais un rôle majeur, non seulement dans la compétitivité des entreprises, mais aussi et surtout dans la diffusion instantanée des informations à grande échelle dans lesquelles les crises sont traitées à ciel ouvert. Aussi, internet et l'ensemble de la téléphonie mobile ont-ils un impact sur les habitudes de communication des Ivoiriens à travers les "médias sociaux ou réseaux sociaux", une terminologie qui semble se décrire sur fond de polémique. Les avis sont partagés sur ces deux termes. Il apparaît donc opportun de les élucider.

Pour F. Charest, «Aux États-Unis comme au Canada (...) sont plus volontiers adoptés les termes "médias sociaux", tandis que les professionnels et les universitaires européens optent pour les termes "réseaux sociaux" "(2012, pp.31-50). Dans les deux cas de figure, il s'agit d'interconnexion entre des personnes, des groupes de personnes ou encore des entités 
sociales qui interagissent ensemble. Mais pour Antoine Dupin, spécialiste du numérique, une distinction doit être faite entre "médias sociaux et réseaux sociaux". En effet, pour lui,

«Les médias sociaux proposent une interaction sociale qui se fonde sur la création et l'échange des contenus et les réseaux sociaux se reposent sur un lien social qui rassemble les personnes (...). Les réseaux sociaux (Facebook, Linkedin) sont une partie des médias sociaux à l'image des blogs, Twitter, YouTube, Dailymotion, etc. » (2010).

Dans cette perspective, l'auteur précise sa pensée en indiquant

«...Qu'ils regroupent les technologies, les applications et les sites permettant aux utilisateurs d'être facilement producteurs de contenus et d'interagir rapidement et à distance sous l'étiquette de "médias sociaux numériques" » (idem,2010).

17 La présente réflexion reprend à son compte, sans réveiller la polémique, la terminologie de "médias sociaux numériques" pour désigner l'ensemble des technologies médiatiques. Il s'agit en d'autres termes, de présenter l'arsenal innovant des moyens et des outils de communication permettant la production et la diffusion à distance, par des professionnels, mais également par des amateurs, du flux d'informations, de transmissions de photos, des images, des films, des vidéos, etc., en dehors des médias traditionnels.

Ainsi sans être exhaustifs, sont exposés à titre illustratif, les principaux médias sociaux numériques à savoir Linkedin, Twitter, YouTube, Dailymotion, Facebook, les blogs, etc., qui sont des espaces de prise de parole formelle ou informelle dans lesquels les internautes défendent librement une cause ou un secteur d'activité. Cette exploration définitionnelle permet maintenant de mieux envisager l'analyse de l'impact des médias numériques dans l'attaque de Grand-Bassam.

\section{De la révolution des TIC à l'impact des médias numériques dans l'attaque de Bassam}

Il convient d'indiquer que les TIC ont connu, au cours de la dernière décennie, une expansion remarquable en Côte d'Ivoire. "Sa croissance soutenue, de plus de $10 \%$ par an, permet à ce secteur de contribuer aujourd'hui à hauteur de $6 \%$ du PIB $»^{3}$. Cependant, cette performance reste marginale quand on note le fossé numérique grandissant entre le Nord et le Sud.

La vulgarisation des ordinateurs et la généralisation des ordinateurs portables en Côte d'Ivoire ont contribué au développement de l'internet grâce à une relative accessibilité sur le territoire national. De plus, avec l'avènement des Smartphones, la téléphonie cellulaire et toutes les applications qui lui sont liées, tout individu peut facilement produire des images et les véhiculer à un destinataire.

21 A ce sujet, Daniel Kablan Duncan, Premier ministre du gouvernement ivoirien, disait qu' "en Côte d'Ivoire, nous avons 24 millions d'habitants avec au moins 24 millions d'abonnés au téléphone portable $"^{4}$. Force est de constater que, du simple vendeur de rue à l'ouvrier peu qualifié jusqu' au cadre supérieur ou au directeur de société, chaque Ivoirien, détenteur de ces moyens technologiques, les manipule à souhait en temps réel et pour des raisons diverses : envoyer et recevoir des mails et des Short Message Service (SMS), faire des prises de vue, écouter de la musique ou des informations à la radio, etc. 
22 En outre, ces technologies ont engendré la production, en grande série, des informations qui font d'elles des produits de masses. Les réseaux sociaux y ont fait leur entrée si bien qu'aucune contrée de la planète n'est à l'abri de leur impact. L'on dénote parfois un usage mimétique des produits issus des TIC qui semblent être à la portée de tous les citoyens.

On a appelé les technologies médiatiques, l'ensemble des médias en interconnexion empruntant de nouveaux moyens dans la transmission instantanée de l'information. Dans ce cadre, il faut indiquer, outre les professionnels, qu'il existe également des amateurs qui se forgent au métier de journalisme occasionnel à travers les réseaux sociaux alimentés par Facebook, Twitter, etc., qui ont véhiculé de vraies ou fausses nouvelles sous forme de rumeurs qui envahissent les rédactions ou les inondent de photos parfois horribles dénuées de toute valeur éthique ou déontologique.

Ainsi, dès les premières heures de l'éclatement de la crise, devant les bruits de détonations qui ont été entendus, certains internautes et des personnes anonymes ont "tiré la sonnette d'alarme" en donnant les premières informations. Ils ont échangé rapidement des SMS avec des correspondants pour dire que les détonations entendues provenaient des bandits qui ont essuyé des tirs alors qu'ils s'attaquaient aux restaurants en bordure de plage à Grand-Bassam; d'autres faisaient allusion à de simples pétards comme le révèle un témoin : "Nous étions à 200 mètres des lieux et nous avons entendu des coups de feu... Certains faisaient allusion à des pétards $»^{5}$.

Les premières informations, parfois teintées de rumeurs, envoyées sans vérification par des internautes amateurs, ont fait place, aux environs de 12 heures, à des informations plus fines des agences telles que l'Agence France Presse (AFP), l'Agence Ivoirienne de Presse (AIP) et Radio France Internationale (RFI), etc., qui ont envoyé à leurs abonnés du service news des informations instantanées sous forme de SMS sur leur téléphone portable : "Des tirs nourris ont été entendus dans un hôtel à Gand-Bassam et font état d'attaque terroriste».

26 Au fur et à mesure que le temps s'écoulait, les informations parvenaient aux téléphones portables et elles se précisaient davantage. Ainsi, les internautes, usagers de téléphones mobiles et toutes les parties prenantes des médias numériques participaient à l'événement de la crise en envoyant les photos des victimes sur Facebook. Les médias traditionnels, pendant ce temps, n'avaient pas encore pu recouper toutes leurs informations pour les diffuser. Et pourtant, toute la Côte d'Ivoire était inondée d'informations sur l'attaque terroriste de Grand-Bassam grâce à la force du numérique, mais surtout grâce au fait que les récepteurs avaient pris l'habitude de s'approprier l'outil internet, les téléphones mobiles et ses différentes applications. Au cours de l'étude, certains enquêtés ont souligné avoir filmé et fait vivre en direct, à partir de leurs téléphones portables, les événements de l'attaque à des correspondants situés à plusieurs centaines de kilomètres de Grand-Bassam :

«(...) Nous avons mis à contribution nos téléphones portables pour informer nos parents et amis dans les premières heures d'abord...Ensuite, quand il y a eu une accalmie, nous avons fait des photos et des vidéos des lieux et de quelques victimes pour nos proches... ${ }^{6}$ "

En dépit de cette kyrielle d'informations déversées sur la Côte d'Ivoire par internet, les téléphonies cellulaires, les chaînes d'informations étrangères, la Radio Télévision Ivoirienne (RTI), média traditionnel d'information gouvernemental, était restée paradoxalement muette toute la journée sur l'événement. Un magazine ivoirien, people "VIP MAG" exposait à sa "Une", un mois après cette triste attaque que 
« Les Ivoiriens étaient en colère de n'avoir aucune information sur ce qui se passe à Bassam à travers leur radio et télé... Seuls les réseaux sociaux et certaines chaînes de radio et de télé étrangères leur donnaient des bribes d'informations... " " crucial de la crise, a fait dire à plus d'un observateur avisé qu'une crise d'information se dessinait au cœur du pouvoir ivoirien dans la gestion de l'attaque de Grand-Bassam. N'est-ce pas là un signe annonciateur de la façon dont le gouvernement entendait gérer l'attaque sur le plan de la communication?

\section{De l'attaque de Grand Bassam à la crise de communication gouvernementale}

\section{La gestion de la communication gouvernementale de crise}

Compte tenu de l'urgence, de l'irruption des parties prenantes sur la scène, du stress engendré et du traumatisme causé par la crise, le gouvernement ivoirien, appelé sur tous les fronts, était contraint de réagir rapidement dans l'objectif d'apaiser la population et d'éteindre les feux de la crise. Le gouvernement voulait prendre l'ampleur de l'événement alors qu'il maîtrisait insuffisamment les différents contours de l'information sur le nombre exact des victimes, les mobiles et les circonstances de l'attaque. Dès lors, les déclarations gouvernementales étaient mises à l'épreuve du terrain. La population qui était avide d'informations, s'était fait une idée des échos déjà reçus des réseaux sociaux et des chaînes étrangères alors que, comme annoncé précédemment, la RTI, la chaîne nationale, apparaissait silencieuse au moment où beaucoup d'Ivoiriens pensaient à juste titre qu'elle devrait donner de la voix, à savoir, proposer, par exemple, entre autres, une bande déroulante d'informations, un flash d'informations ou même un direct de plateau sur l'attaque de Grand-Bassam.

Contrairement à toutes ces propositions, la RTI avait préféré diffuser un match de football dans l'après-midi. Les internautes avaient sévèrement critiqué cette absence de couverture médiatique de l'attaque. Il est opportun de résumer, dans le cadre de cette étude, leur point de vue en ces termes :

« La RTI n'a jamais été au cœur des événements, voilà pourquoi nous nous tournons plus vers les réseaux sociaux, les chaînes étrangères comme France 24 et autres. la RTI a toujours eu une longueur de retard sur les autres (...). Je comprends maintenant que la RTI ne travaille pas pour le peuple ; il y a des morts et la RTI fait croire que tout va bien. Devant son silence face à l'attaque, je peux conclure qu'elle fait du terrorisme informationnel... $»^{8}$

31 En matière de communication de crise, le critère de temps est un facteur prépondérant pour maîtriser celle-ci. Dans le cadre de cette étude, le constat montre qu'il y a eu un long silence entre le moment de l'attaque et la réaction du gouvernement si bien que les réseaux sociaux ont eu le temps d'occuper le terrain. Ainsi des survivants aux simples spectateurs de l'attaque avec leurs propres moyens (téléphones portables, internet, réseaux sociaux, etc.) ont relayé eux-mêmes l'information auprès des correspondants, des parents, des amis et des connaissances : "Un long silence... Près de cinq heures d'intervalle entre les premiers tirs ( $12 \mathrm{~h}$ 25) et la déclaration à la télévision nationale du ministre de l'Intérieur, Hamed Bakayoko $(17 \mathrm{~h} 10)^{9}$ ». Le premier reportage consacré aux événements ne sera diffusé que peu avant 20 heures par la RTI, dans lequel le gouvernement fait une déclaration, nourrie de confusion, faisant état de six terroristes neutralisés. Par la suite, l'on note des 
contre-vérités dans la production des communiqués traduits en ces termes: «...Puis la publication d'un premier communiqué gouvernemental très flou et vite retiré; un bilan faisant état de six terroristes "neutralisés", avant d'être réduit à trois... $»^{10}$.

Autrement dit, les premiers signes de balbutiements avaient fait jour avec le peu d'assurance dans l'annonce du nombre des victimes. Ceci était devenu un sujet à controverse au sein de la population. D'ailleurs, beaucoup de personnes avaient soutenu la version suivante :

"Certains témoins ne se lassent pas de relater ce qu'ils ont "vécu en direct" et beaucoup assurent avoir vu plus de trois assaillants, contredisant la version officielle qui fait état de trois "terroristes abattus" en plus des 15 civils et trois membres des forces de sécurité morts dans l'attaque $»^{11}$.

La gestion de la crise dépendait du comité de crise mis en place. Le président Alassane Ouattara s'est entretenu très vite par téléphone avec ses plus proches collaborateurs civils et militaires afin de recouper toutes les informations. Les questions se bousculaient et les réponses, en provenance de son premier cercle décisionnel, étaient parfois diamétralement opposées. Le ministre de l'intérieur, Hamed Bakayoko, qui était rentré du Ghana, se joint au Poste de Commandement (PC) installé au sein de son ministère. Sur le terrain, un PC est également installé à l'hôtel de France de Grand- Bassam et selon Jeune Afrique :

«(...) C'est à Assinie, une station balnéaire située à $40 \mathrm{~km}$ de Grand-Bassam, que toutes les décisions seront prises. À $14 \mathrm{~h} \mathrm{45}$, endossant alors ses habits de chef suprême des armées, Alassane Ouattara ordonne l'assaut. Ce n'est qu'à la fin de celui-ci qu'il sera héliporté à Abidjan. Une heure et demie plus tard, il se rend à Grand-Bassam ${ }^{12}$ ».

Des mesures de sécurité avaient été prises aux abords des hôtels et des centres commerciaux d'Abidjan. Les préfets avaient été mis en état d'alerte. La gestion insuffisante de la crise avec ses corollaires d'imprécisions dans la collecte et la diffusion des informations du terrain se posent en tant qu'enjeux de la réception de l'information gouvernementale. C'est à juste raison que le quotidien Nord Sud, s'inscrivant dans la guerre des chiffres, est revenu sur une adresse faite par le ministre Hamed Bakayoko deux jours après l'attaque : «Le chiffre des assaillants tués a baissé de 3 terroristes au lieu de six abattus dont les corps se trouvent à la morgue $»^{13}$. Cette information gouvernementale, de première main, véhiculée a été perçue différemment par la population qui a su créer ellemême une source parallèle d'information, grâce aux réseaux sociaux.

Une analyse de l'impact psychosociologique sur la population au lendemain de l'attaque présente que toutes les couches sociopolitiques et religieuses ont été marquées par une solidarité agissante face à la crise. Il est à noter que des partis politiques tels que le PDCI et le RDR deux partis alliés au pouvoir, le FPI se présentant comme le chef de file de l'opposition, le Conseil Supérieur des Imams en Côte d'Ivoire (COSIM) représentant la communauté musulmane, la Conférence des Evêques Catholiques de Côte d'Ivoire, une organisation de l'Église catholique, le conseil Supérieur des Rois et chefs traditionnels de Côte d'Ivoire qui a un poids socioculturel prépondérant dans la prise décision des décideurs politiques, etc. ont unanimement condamné cette attaque terroriste de GrandBassam. En outre, ces organisations, en déplorant dans leur déclaration cette attaque, elles ont manifesté leur compassion, leur proximité en faveur de tous les compatriotes, des blessés et de toutes les victimes,

«...Le RDR s'incline devant la mémoire des morts de cette barbarie (...), condamne cet acte irresponsable (...).Pour le COSIM, en islam, la vie est sacrée...Il condamne 
avec la dernière énergie, ces ignobles assassinats (...).Le FPI et Ouattara se sont entretenus : Affi, président du FPI, affirme qu'aucune autorité digne de ce nom, ne peut rester insensible face à ce carnage... Nous témoignons notre solidarité aux familles des victimes, aux blessés et aux autorités... » ${ }^{14}$.

Cette mobilisation exceptionnelle de toutes les forces vives de la mère patrie a contribué à apaiser les tensions politiques. L'ennemi commun semble être l'attaque de GrandBassam dont la gestion communicationnelle a laissé entrevoir quelques failles. Face à cette carence du gouvernement en matière de communication de crise, quelles solutions peut-on proposer et explorer?

\section{Quelques esquisses de solutions à explorer}

En dernière instance, il est à noter qu'une telle communication de crise qui se présente en dents de scie nécessite des stratégies proactives et réactives à même de faire tomber la fièvre de la crise.

D'abord, l'élaboration d'une cellule de crise est un acte anticipatoire nécessaire relevant de veille stratégique et non de l'improvisation sujette à la cacophonie. En d'autres termes, une telle cellule de crise, hâtivement constituée avec des parties prenantes en son sein, at-elle des habilités à traiter des questions de crises ? S'est-elle exercée quotidiennement pour être outillée à contrer toute crise? Son rôle premier, dans le concept de la proactivité, est d'identifier les pistes possibles susceptibles de résoudre les problèmes repérés et de proposer des solutions alternatives ou des pistes de recherche.

Des simulations et des confrontations des points de vue à des crises virtuelles ou crises similaires ayant affecté des pays, constituent des exercices pertinents pour lui permettre d'avoir une réaction appropriée pendant le déclenchement réel de la crise. Ainsi la formation de la cellule outillée pour, d'une part, la gestion du stress, des relations avec les médias, et, d'autre part, pour la préparation des documents d'intervention, et pour l'identification avec les relais d'opinion devront compléter le travail d'anticipation.

C'est dans cette optique qu'est mise en œuvre la stratégie réactive qui consiste à intervenir au bon moment. En effet, la capacité de réaction de la cellule devra être à la hauteur de l'événement en communiquant plus rapidement : «Il faudra communiquer au plus vite pour neutraliser les rumeurs, dépassionner le débat et situer les faits: les causes de la crise, les conséquences effectives et les issues probables... » ( Libaert, Westphalen, 2011, p. 66.).

La prise de conscience de la réduction de la durée du temps de réaction est un remède qui permet de mieux communiquer afin de dissiper les inquiétudes de la population. La préparation par simulations avec une cellule ad hoc et un retour d'expérience, version " corporate » vise à faire acquérir aux managers le réflexe idoine pendant la période critique, attitude nécessaire pour ne pas se laisser submerger par la crise. Ce réflexe permet d'engager une réflexion sur la riposte et de pouvoir enfin conduire l'action.

Sans apprentissage réitéré, il est hautement improbable que l'on puisse réunir les capacités indispensables pour endiguer une crise. Pour n'avoir pas su anticiper, la Côte d'Ivoire a vécu l'expérience de cette crise à ses dépens, une crise qui n'arrive pas seulement aux autres. 


\section{Conclusion}

43 Grand- Bassam. On note, en effet, l'usage des moyens technologiques numériques dans la conception, la transmission et la diffusion des informations par les acteurs de la crise sur le théâtre des opérations. De plus, l'étude a présenté en filigrane une analyse des limites de la gestion communicationnelle de crise du gouvernement à travers ses nombreuses hésitations dans le décompte des victimes pendant la diffusion de l'information auprès de la population. En d'autres termes, il est à indiquer une inadéquation entre l'image qu'a voulu véhiculer le gouvernement ivoirien pendant la crise et celle perçue par le peuple, déjà outillé dans l'usage et l'utilisation de l'internet, de la téléphonie mobile et de ses applications. Finalement, cette appropriation des technologies médiatiques par la population a eu pour corollaire son aisance dans la production et la diffusion des informations numériques pendant la crise terroriste qui a souillé la terre de GrandBassam. Il y a également à noter la mobilité des usagers avides d'informations, leurs « Vaet-vient " sur les chaînes de radio et de télévision étrangères pendant que la chaîne nationale, la RTI, a volontairement refusé de couvrir cet événement de niveau international.

Il n'est pas vain d'indiquer que cette crise a impulsé un élan de solidarité nationale et internationale et les Ivoiriens ont rivalisé d'initiatives sur le plan du numérique. Ainsi sur les réseaux sociaux, on pouvait lire des «posts" du genre «même pas peur, je suis Bassam...». C'est dans cet élan que les pratiques traditionnelles, qui relèvent des représentations socioculturelles, ont pris le relais des ballets diplomatiques en visites des sites, pour "purifier" la terre de Grand-Bassam, station balnéaire ivoirienne "souillée" par l'attentat jihadiste du 13 mars 2016, qui contrastent certes, avec le contexte de modernité. Sur le plan socio-anthropologique, l'on dénote une convergence de point de vue des leaders politiques allant dans le sens de l'apaisement. La plupart des Ivoiriens, le cœur meurtri, ont marqué leur solidarité et leur compassion face à l'épreuve, à l'instar du gouvernement qui a fait un geste pour soulager financièrement les victimes et les différents dons octroyés par la communauté internationale comme en témoigne le communiqué du conseil des ministres :

«Un montant total de 300 millions de Francs CFA sera décaissé pour soutenir la ville de Grand-Bassam, (...). Les présidents du Togo, Faure Gnassingbé, et du Bénin, Thomas Boni Yayi, ont fait chacun un don de 50 millions à la Côte d'Ivoire (...). La France a fait un don de médicaments d'une valeur de 150 millions de F CFA.... ${ }^{15}$.

Face à l'épreuve, cet élan de solidarité dégagé par la population ivoirienne et relayé toujours par les réseaux sociaux, devrait être soutenu par le gouvernement à travers une communication sociale axée sur la sensibilisation, l'éducation aux médias et de la masse concernant les effets du terrorisme. 


\section{BIBLIOGRAPHIE}

Martial Alate, « La mauvaise gestion de l'attaque de Grand-Bassam par la RTI ; la ministre et la nièce d'Ado accusées » VIP MAG, N 077, du 12 au 15 mai 2016, p. 2.

Mieu Baudelaire, Niakate Haby, «Attentat de Grand-Bassam : sept questions pour comprendre », Jeune Afrique $\mathrm{N}^{\circ} 2880,20$ mars 2016.

Zoumana Coulibaly, « Eugène Dié Kacou, témoin oculaire », in Patriote, $\mathrm{N}^{\circ}$ 4878, 14 mars 2016, p. 4. Jean-Michel Plane, Théorie des organisations, Paris, Dunod, 4e édition, 2014.

Thierry Libaert, Marie-Hélène Westphalen, La communication externe des entreprises, Paris, Dunod, 3e édition, 2011.

Thierry Libaert, La Communication de Crise, Paris, Dunod, Les Topos, 2001.

Alexis Noumé, Nouvelles Technologies. « Le numérique, un levier de croissance pour les PME » in Côte d'Ivoire Economie, http://news.abidjan.net/h/588515.html, Publié le lundi 4 avril 2016.

Bè Ousmane Tenin, « Attaque terroriste de Grand-Bassam / Hamed Bakayoko, ministre de l'Intérieur et de la sécurité : "Des personnes ont été arrêtées », Nord Sud Quotidien, N 3199, Mardi 15 mars 2016, p, 4

Joan Woodward, Industrial Organization. Theory and practice, Oxford University Press, Oxford, 1965.

\section{NOTES}

1. Koudou, Claude. «Attaque de Grand- Bassam : des coïncidences troublantes et des déclarations gouvernementales contradictoires. Que nous cache-t-on?» in https://www.ivoirebusiness.net/ articles/attaque-de-grand-bassam-des-coincidences-troublantes-et-des-declarationsgouvernementales, Publié le mercredi 16 mars 2016.

2. Nouvelles technologies : Le numérique, un levier de croissance pour les PME Publié le lundi 4 avril 2016, Côte d'Ivoire Economie http://news.abidjan.net/h/588515.html.

3. Op-cit.

4. Daniel Duncan,Premier, Conférence de presse du vendredi 13 mai 2016, diffusée par la RTI relative au coût élevé de l'électricité en Côte d'Ivoire

5. Coulibaly, Zoumana. «Eugène Dié Kacou, témoin oculaire ", in Patriote, $\mathrm{N}^{\circ}$ 4878, lundi 14 mars 2016, p. 4

6. Enquête qualitative menée par Toa Jules le 17 mars 2016 à Grand-Bassam auprès d'un groupe de personnes en vue de recueillir leurs opinions sur l'impact du numérique dans la transmission et la réception des informations lors de l'attaque de Grand-Bassam.

7. Martial, Alaté. « La mauvaise gestion de l'attaque de Grand-Bassam par la RTI ; la ministre et la nièce d'Ado accusées » VIP MAG, $\mathrm{N}^{\circ}$ 077, du 12 au 15 mai 2016, p. 2.

8. http://news.educarriere.ci/news-17489-at-taques-de-gand-bassam-la-rti-au-coeur-de-lapolemique.html, https://www.Facebook.com/djiba.diabate et de http://www.facebook.com/ serges.asseu

9. Baudelaire, Mieu, Haby Niakate. Attentat de Grand-Bassam : sept questions pour comprendre la crise, $\mathrm{N}^{\circ} 2880$ de Jeune Afrique publié le 20 mars 2016 à 09 h43. 
10. Baudelaire, Mieu, Haby, Niakate, Op-Cit.

11. Evelyne AKA. Tourisme Côte d'Ivoire attentat jihadistes14 mars 201619 : 32 par l'AFP.

12. Baudelaire, Mieu Haby, Niakate. Idem.

13. Ténin, Bè Ousmane. "Attaque terroriste de Grand-Bassam / Hamed Bakayoko, ministre de l'Intérieur et de la sécurité : "Des personnes ont été arrêtées », Nord Sud Quotidien, Nº 3199, Mardi 15 mars 2016, p, 4.

14. «Attaque terroriste de Grand-Bassam » in Notre Voie $\mathrm{N}^{\circ}$ 5255, mardi 15 mars 2016.

15. Tweet de [at] Gouvci, 16 mars 2016 à 16 h 10 - Mis à jour le 16 mars 2016 à 17 h05.

\section{RÉSUMÉS}

Les technologies médiatiques, dans cette réflexion, sont comprises comme l'ensemble de l'arsenal innovant des moyens et des outils de communication, en dehors des médias traditionnels. Cette étude, sous l'angle de la théorie des représentations et dans le cadre épistémologique de la socio- anthropologie, analyse l'impact de ces technologies dans le traitement de la crise survenue le 13 mars 2016 à Grand-Bassam, suite à l'attaque criminelle des assaillants djihadistes. En effet, grâce aux TIC et leurs multiples applications, la production en grande série d'informations sur la crise a été diffusée à travers les réseaux sociaux. Ce qui dénote leur impact réel qui a, d'ailleurs, contraint le gouvernement ivoirien à mener une communication de crise lors des événements de Grand-Bassam.

Media technologies, in this reflection, are understood as all of the innovative arsenal of means and tools of communication, outside of traditional media. The study, from the perspective of the theory of representations and in the epistemological framework of anthropology, analyzes the impact of these technologies in the treatment of the crisis occurred on March 13th, 2016 at Grand- Bassam, following the attack criminal jihadist attackers. Indeed, thanks to ICTs and their multiple applications, the mass production of information on the crisis was diffused through social networks. This denotes their real impact has, however, forced the Ivorian government to conduct a crisis communication at Grand- Bassam events

Tecnologías de la comunicación, en esta reflexión, se entienden como todo el arsenal innovadora de los medios y herramientas de comunicación, fuera de los medios tradicionales. Este estudio, en términos de la teoría de la representación y el marco epistemológico de la socio-antropología, analiza el impacto de estas tecnologías en el tratamiento de la crisis de marzo de 13 Se ha producido, 2016 Grand-Bassam, siguiendo el ataque criminal atacantes jihadistas. De hecho, gracias a las TIC y sus múltiples aplicaciones, la producción masiva de información sobre la crisis se difundió a través de redes sociales. Esto denota el impacto real tiene, sin embargo, obligado al gobierno de Côte d'Ivoir para llevar a cabo una comunicación de crisis en los eventos de Grand Bassam. 
INDEX

Keywords : technology, media, social networks, crisis in Grand-Bassam

Palabras claves : tecnología, medios, redes sociales, crisis en Grand-Bassam

Mots-clés : technologies, médias, réseaux sociaux, crise à Grand-Bassam

\section{AUTEUR}

JULES EVARISTE AGNINI TOA

Université Félix Houphouët-Boigny, Côte d'Ivoire 